\title{
CAN ONE REALLY STUDY CHAOS ANALYTICALLY?
}

\author{
M. HOWARD LEE ${ }^{a, b}$ \\ ${ }^{a}$ Department of Physics and Astronomy, University of Georgia, Athens, GA 30602 USA \\ ${ }^{b}$ Korea Institute for Advanced Study, Seoul 130-012, Korea \\ correspondence: MHLee@uga.edu
}

ABSTRACT. One generally thinks that chaos can be studied only numerically by aid of the computer. It is however suggested by the theorem of Sharkovskii and $\mathrm{Li}$ and Yorke that in Id continuous maps analytical studies are possible. How one might achieve such a goal in one special map is described.

KEYWORDS: chaos, logistic map, Sharkovskii theorem.

\section{INTRODUCTION}

When one thinks of chaos, what most likely come to mind are beautiful pictures drawn by the computer. In fact virtually all articles and books on chaos have some such pictures illustrating unimagined properties of nonlinear behavior $[1,2]$. Thus one tends to think that an analytical approach to chaos is nearly unthinkable. One might even ask why one should want to attempt it. If it were, however, possible even in a special case or cases, it could help provide perhaps a deeper understanding. This attitude is to our mind something that we do or try to do in physics.

Such a possibility is opened by a remarkable theorem proved by Sharkovskii [3], and later independently by Li and Yorke [4. This theorem applies to one-dimensional continuous maps of real numbers in a finite interval. The essence of the theorem reads that if 3-cycle exists in one domain of such a map, it implies the existence of all other cycles in the same domain and hence it implies the existence of chaos itself therein. What is especially significant, and not yet widely recognized, is that by means of 3-cycle a chaotic domain can be determined without introducing a working definition of chaos. This is contrary to what is normally done in numerical studies. In these studies, a domain is said to be chaotic if it meets a certain criterion or a set of criteria, which represent working definitions of chaos. These definitions are phenomenologically based, not derived from some higher principles of chaos.

We will not go into why 3-cycle plays this pivotal role, for this subject is now well known. What we will do is to demonstrate the existence of 3-cycle in an applicable map and how we can learn about chaos from the content of the theorem. This article being intended as an overview, we will not present any detailed derivations, just ideas of what can be done.

\section{ONE-DIMENSIONAL CONTINUOUS MAP OF REAL NUMBERS IN AN INTERVAL}

Let $x_{i}$ and $x_{j}$ be two real numbers in $(0,1)$. If $x_{i} \rightarrow x_{j}$ by an action $f$, we write the action as $x_{j}=f\left(x_{i}\right)$, calling it a map. If, for some value of $x_{i}$, say $x *$, it goes into itself and goes nowhere else, it is termed a fixed point of $f$, i.e., $f(x *)=x *$. To a fixed point, $\mathrm{f}$ acts like an identity operation.

Let $x_{1}, x_{2}, x_{3}, .$. be a set of points in $(0,1)$, not necessarily ordered. By the action of $f$, let $f\left(x_{1}\right)=x_{2}$, $f\left(x_{2}\right)=x_{3}, \ldots, f\left(x_{N}\right)=x_{N+1}$ etc., a process termed an iteration. Suppose $f\left(x_{3}\right)=x_{1}$, i.e., $x_{4}=x_{1}$. The iteration has terminated in 3 steps giving the 3 -cycle condition: $f\left(x_{1}\right)=x_{2}, f\left(x_{2}\right)=x_{3}$, and $f\left(x_{3}\right)=x_{1}$, which may be expressed as $f(f(f(x))) \equiv f^{3}(x)=x$, where $x=x_{1}, x_{2}$, or $x_{3}$, and $x_{1} \neq x_{2} \neq x_{3}$. These three values are fixed points of $f^{3}$, giving 3 -cycle. Excluded are fixed points of $\mathrm{f}$, which are also fixed points of $f^{3}$, since they do not satisfy the 3-cycle condition.

If $f\left(x_{N}\right)=x_{1}$, it defines $N$-cycle. It would be termed periodic since, like an orbital motion, it returns to its initial point after $N$ steps. If, however, $f\left(x_{N}\right)=$ $x_{N+1}, N \rightarrow \infty$, never returning to its initial point, it would be chaotic, like a trajectory which never closes. Even at this stage, one might ask whether there are some properties of the interval of points, to which the two fundamentally different kinds of trajectory could be ascribed. A periodic trajectory has a finite set of "frequencies". They are probably connected one with another through a sequence of rational numbers that fill the interval. A chaotic trajectory goes from one point to another in a finite interval unpredictably and never returning. This kind of behavior suggests that to describe its movement one would have to bring in the irrational numbers that fill the interval.

Returning to the theorem of Sharkovskii and LiYorke, what is required is to obtain the 3 fixed points of 3-cycle for a particular $1 \mathrm{~d}$ continuous map. It amounts to solving for three roots of $f^{3}(x)-x=$ 0 , probably a cubic equation, which should pose no special difficulty. Had the theorem called for 4-cycle, presenting a quartic equation, it would still be no challenge. Had it asked for 5-cycle, any attempt at an analytical solution would have to be abandoned. Fortuitously it is 3-cycle that the theorem asks for and this is why an analytical study seems feasible.

But we cannot know for sure until the 3-cycle of an applicable map is constructed. We shall now turn to one such map to see what is to be realized. 


\section{LOGISTIC MAP}

Perhaps one of the simplest and no doubt the most widely studied among all chaotic maps is the logistic map. Its widespread appeal recalls the Ising model of magnetism in statistical mechanics. Perhaps it is not too far-fetched to call the logistic map the Ising model of chaos.

The logistic map is a one-dimensional continuous map of real numbers in the interval $(0,1)$, to which the theorem of Sharkovskii is applicable. The usual way to define the map is as $x^{\prime}=f(x)$, where

$$
f(x)=a x(1-x), \quad x=(0,1),
$$

where $a$ is a control parameter, $0 \leq a \leq 4$. The fixed points of $f$ and $f^{2}$ for (1) are obtained readily: By solving $f(x)-x=0$, we obtain $x=x_{0}=1-a^{-1}$, $a>1$. Next by solving $\left(f^{2}-x\right) /(f-x)=0$, we obtain a quadratic equation, from which $x=x_{1,2}=$ $(a+1) / 2 a\{1 \pm \sqrt{(a-3) /(a+1)}\}, a>3$. Now we anticipate that $\left(f^{3}-x\right) /(f-x)=0$ is a cubic equation, from which 3 roots are to be found.

We now construct $Q(x) \equiv\left(f^{3}-x\right) /(f-x)=0$ in a new variable $t=a x$ for simplification [5]:

$$
\begin{aligned}
& Q(t)=t^{6}-(3 a+1) t^{5}+\left(3 a^{2}+4 a+1\right) t^{4} \\
& -\left(a^{3}+5 a^{2}+3 a+1\right) t^{3}+\left(2 a^{3}+3 a^{2}+3 a+1\right) t^{2} \\
& \quad-\left(a^{3}+2 a^{2}+2 a+1\right) t+\left(a^{2}+a+1\right) .
\end{aligned}
$$

To our surprise it is a sextic equation. Since there are no known formulas for solving any equations of degrees equal to or higher than 5 , at a first glance we seem to have arrived at a brick wall.

Perhaps $Q(t)$ is not a general sextic equation but a special one. If so, it might be solvable in some special way. If $E_{k}$ denotes an equation of degree $k$, $E_{6}$ would be solvable at least in principle if it could be expressed as: $E_{2} \times E_{2} \times E_{2}$ or $E_{3} \times E_{3}$. Looking at (2), we see at once that $Q(t)$ must be real if $\mathrm{t}$ is real since its coefficients are all real. This means that the 6 roots can only be either 3 pairs of complex conjugates or two sets of 3 real roots, corresponding to two solvable forms for $Q(t)$. This impels us to look for an indication that $Q(t)$ is not general. If (2) is expressed as

$$
Q(t)=\sum_{k=0}^{6}(-)^{k} \delta_{k} t^{k}
$$

we find that $\Delta \equiv \sum_{k=0}^{5}(-)^{k} \delta_{k}=0$, referred to as the delta sum rule. This sum rule is a clear indication that $Q(t)$ is not a general sextic equation.

\section{Solving the SeXtic EQUATIOn: PRELIMINARIES}

If $a=0$ in (2), which still satisfies the delta sum rule, the roots are: $\exp ( \pm i \pi / 7), \exp ( \pm i 3 \pi / 7)$ and $\exp ( \pm i 5 \pi / 7), 3$ pairs of complex conjugates lying on the unit circle. If the roots are to be real, none of them may lie on the negative real axis of $t$ since the coefficients of $t$ in odd power have '-' signs. The six roots must all lie on the positive real axis. If $a \rightarrow \infty$, one of the roots goes as $1 / a$ asymptotically. Thus as $a$ increases from zero, the three pairs of complex roots must move toward the positive real axis. At some value of $a=\tilde{a}$ say, each complex-conjugate pair becomes two identical real roots simultaneously. Thereafter each degenerate real root splits into two real roots. This overall behavior is consistent with the two solvable forms for $Q(t)$. We cannot yet know however whether $\tilde{a}<4$ or $>4$. The existence of 3 -cycle in the logistic map requires that $\tilde{a}$ be less than 4 and also that at least one set of real roots lies in the interval $t=(0, a)$ or $x=(0,1)$. If otherwise, 3 -cycle does not exist in it.

If $a>\tilde{a}$, there must be two sets of 3 positive real roots. Hence, we shall write $Q(t)=q(t) \times q^{\prime}(t)$, where $q$ and $q^{\prime}$ are cubic equations with (as yet undetermined) roots, resp., $t_{k}$ and $t_{k}^{\prime}, k=1,2,3$. Thus one can express $q(t)$ as

$$
q(t)=t^{3}-\alpha t^{2}+\beta t-\gamma,
$$

where $\alpha=t_{1}+t_{2}+t_{3}, \beta=t_{1} t_{2}+t_{2} t_{3}+t_{3} t_{1}, \gamma=t_{1} t_{2} t_{3}$, and $q^{\prime}(t)$ similarly with primes written on. They $(\alpha \beta \gamma)$ will be referred to as trigonals.

To understand why $Q$ is special we look for some structural relations between the trigonals of the same kind contained in the structure of the logistic map. Indeed we find that [7]: $\alpha-\beta+\gamma=0$ and $\alpha^{\prime}-\beta^{\prime}+\gamma^{\prime}=$ 0 , together called the trigonal relation. To no surprise the trigonal relation implies the delta sum rule. In addition, $\beta=(a+1) \alpha-\left(a^{2}+a+1\right)$ and $\gamma=a \alpha-\left(a^{2}+\right.$ $a+1)$, leaving only $\alpha$ and $\alpha^{\prime}$ independent, leaving only two unknowns to the problem. The transition condition $E_{2} \times E_{2} \times E_{2}=E_{3} \times E_{3}$, together with the trigonal relation yields $\sigma=0$, where $\sigma=\left(a^{2}-2 a-\right.$ $7)^{1 / 2}$. Hence, $\tilde{a}=1+\sqrt{8}=3.872281323 \cdots$ as the transition value. Since $\tilde{a}<4,3$-cycle can exist in the logistic map.

There are two sets of 3 positive real roots, or two forms of 3-cycle, that had not been implied by the theorem of Sharkovskii, but that evidently exist in the logistic map. Unless their relationship is found, the two cubic equations are still left with one unknown each, $\alpha$ or $\alpha^{\prime}$. How to distinguish the two remains an obstacle to the final solution.

\section{SOlVING THE SEXTIC EQUATION: AN INTERNAL DEGREE OF FREEDOM}

If there are two forms of 3-cycle, they must be distinguishable by some internal degree of freedom in $q$ and $q^{\prime}$. It cannot appear in $Q$, meaning that it cannot appear in $\delta^{\prime} s$, the coefficients of $t$ in $Q$, e.g. $\delta_{5}=3 a+1=\alpha+\alpha^{\prime}$. Since the two forms must be equivalent, they are like two states of parity, 
which could be represented by a double-valued function. For 3-cycle in the logistic map it happens to be $\sigma=\left(a^{2}-2 a-7\right)^{1 / 2}$ [7]. Thus, we shall assume

$$
\begin{aligned}
\alpha & =\frac{1}{2} \delta_{5}+K \sigma, \\
\alpha^{\prime} & =\frac{1}{2} \delta_{5}-K \sigma,
\end{aligned}
$$

where if $K=1 / 2$ it yields all the coefficients $\left(\delta^{\prime} s\right)$ exactly. Therewith we finally obtain:

$$
\begin{aligned}
\alpha & =\frac{1}{2}(3 a+1+\sigma) \\
\beta & =\frac{1}{2}\left(a^{2}+2 a-1+(a+1) \sigma\right), \\
\gamma & =\frac{1}{2}\left(a^{2}-a-2+a \sigma\right),
\end{aligned}
$$

and $\alpha^{\prime}, \beta^{\prime}, \gamma^{\prime}$ by taking $-\sigma$ in $(7)-(9)$. Hence if $q=$ $q(\sigma), q^{\prime}=q(-\sigma)$. It is sufficient to solve only one of them.

Even beforehand, it is possible to prove that $t_{k}$ and $t_{k}^{\prime}, k=1,2,3$, all lie in the interval $(0, a)$. The proof goes as follows: Let $t_{k}=a-\epsilon$. If $\tilde{a} \leq a \leq 4, t_{k}>0$ as already proved. Hence $a-\epsilon>0$. This gives $\epsilon<a$, an upper bound on $\epsilon$. To determine a lower bound on $\epsilon$, we consider the trigonal relation with $t_{k}=a-\epsilon$ for any $k=1,2$ or 3 . By (7)-(9) we find that

$$
\alpha-\beta+\gamma=-R(\epsilon) /(a-\epsilon),
$$

where

$$
R(\epsilon)=\epsilon^{3}-A \epsilon^{2}+B \epsilon-1,
$$

where $A$ and $B$ are real and positive if $\tilde{a} \leq a \leq 4$. The trigonal relation requires that $R(\epsilon)=0$. This is possible if and only if $\epsilon>0$, which gives a lower bound on $\epsilon$. Thus, $0<\epsilon<a$ and this implies that $t_{k}=a-\epsilon$ must lie in the interval $t=(0, a)$. This proof agrees with the cubic solutions obtained from $q=0$ and $q^{\prime}=0$. We conclude that the 3 -cycle exists continuously in the $\operatorname{logistic}$ map from $a=\tilde{a}$ to $a=4$. Throughout this domain there exists chaos because there are, according to the theorem of Sharkovskii, infinitely many cycles of all kinds.

\section{Conclusion: Aleph CyCle And CHAOTIC TRAJECTORIES}

The theorem of Sharkovskii asserts that if 3-cycle exists in a domain, all other cycles exist in that domain. For the logistic map we take this to mean that there are infinitely many cycles of all varieties in the domain $\tilde{a} \leq a \leq 4$. Thus, in this domain the fixed points form a dense set of points in $x=(0,1)$, a strip made up of irrational points of all different kinds. It has a finite measure. If $x_{1}$ is an initial value that belongs to this set of points of finite measure, $f^{N}\left(x_{1}\right)=x_{N+1}, N \rightarrow$ $\infty$, termed an aleph cycle. Evidently an aleph cycle describes a chaotic trajectory. If $x_{0}$ is an initial value that does not belong to this set, thus belongs to a set of points of 0 measure, $f^{M}\left(x_{0}\right)=x_{0}, M<\infty$, a finite cycle, giving a periodic trajectory.

This assertion can be justified for some special values of $a$ such as $a=4$, for which one can obtain a large number of fixed points from which a set of points of finite measure can be constructed [6].

An aleph cycle has a very close connection to an ergodic trajectory in statistical mechanics according to the ergometic theory of the ergodic hypothesis [8]. It is thus possible to gain via an aleph cycle an insight into the ergodic theory of chaos that has been proposed [9].

As we have stated at the outset, while it would be nearly impossible to study chaos analytically, the theorem of Sharkovskii gives a window through which something can be done analytically, as we have described here in this short note.

\section{ACKNOWLEDGEMENTS}

I thank Dr Miloslav Znojil of the Doppler Institute for Mathematical Physics and Applied Mathematics, Rez, Czech Republic for suggesting that I write this article based on a talk presented at Vila Lanna, Prague in October 2013.

\section{REFERENCES}

[1] B. Hu, Phys. Rep. 91, 234 (1982) DOI: $10.1016 / 0370-1573(82) 90057-6$

[2] D. Gulick, Encounters with chaos (McGraw-Hill, NY 1992).

[3] A.N. Sharkovskii, Ukr. Math. Z. 16. 61 (1964).

[4] T-Y Li and J.A. Yorke, Am. Math. Monthly 82, 985 (1975).

[5] M.H. Lee, Acta Phys. Pol. B 42, 1071 (2011).

[6] M.H. Lee, Acta Phys. Pol. B 43,1053 (2012).

[7] M.H. Lee, Acta Phys. Pol. B 44, 925 (2013).

[8] M.H. Lee, Phys. Rev. Lett. 87, 250601 (2001) DOI: 10.1103/PhysRevLett.87.250601; Phys. Rev. Lett. 98, 110403 (2007) DOI: 10.1103/PhysRevLett.98.110403

[9] J.-P. Ekmann and D. Rulle, Rev. Mod. Phys. 57, 617 (1985) DOI: 10.1103/RevModPhys.57.617 